\title{
ON IMMERSIONS WITH SINGULAR SECOND FUNDAMENTAL FORM OPERATORS
}

\author{
EDSEL F. STIEL
}

1. Introduction. In Euclidean 3-dimensional space an isometric immersion is flat if and only if its second fundamental form operators are singular at each point. A similar result holds for hypersurfaces of Euclidean $n$-space, $R^{n}$. However the flat torus can be isometrically immersed in $R^{4}$ in such a way that at any point, almost all of its second fundamental form operators are nonsingular. In fact any compact immersion has this property. This can be seen from the results of [3] which show that the total curvature of such an immersion is always strictly positive while the total curvature is equal to the integral over the manifold of $K^{*}$ where $K^{*}$ at a point can be expressed as the integral of the absolute values of the determinants of the second fundamental form operators. We shall characterize the pointwise behavior of certain immersions with singular second fundamental form operators and from this result describe immersions having this property at every point; i.e. immersions of zero total curvature.

Let $M^{d}$ and $\bar{M}^{d+k}$ denote $C^{\infty}$ Riemannian manifolds and let $\psi$ denote an isometric immersion from $M$ in to $\bar{M}$. The index of relative nullity $\nu$, [2], assigns to each $m$ in $M$ the dimension $\nu(m)$ of the subspace of vectors $x$ in the tangent space $M_{m}$ such that $T_{x}=0$. The linear difference operators $T_{x}$ act on $\bar{M}_{\psi(m)}$ and contain the same information as the second fundamental form operators $S_{z}$ where $z$ is a tangent vector to $\bar{M}$ orthogonal to $d \psi\left(M_{m}\right),[1]$. These operators are characterized by their skew-symmetry and the relations $T_{x}(z)=d \psi\left(S_{z} x\right)$. To simplify notation we shall identify elements of $M_{m}$ and $d \psi\left(M_{m}\right)$ when it seems safe to do so. The immersion $\psi$ is developable at $m$ in $M$ if on all planes $P$ in $M_{m}$ the sectional curvature of $P$ is equal to the sectional curvature of $d \psi(P)$ in $\bar{M}_{\psi(m)}$. We shall later use the result, which follows from the Gauss equation for an immersion, that developability is characterized by the relation $\left\langle T_{x} x, T_{y} y\right\rangle=\left\langle T_{x} y, T_{x} y\right\rangle$ for all $x, y$ in $M_{m}$. By extension of a result in [2] it can be shown that $\nu(m) \geqq d-k$ for an immersion developable at $m$. Thus for $k<d$ it follows that $S_{z}$ is singular for all $z$ in $\bar{M}_{\psi(m)}$ orthogonal to $d \psi\left(M_{m}\right)$. In particular these observations together with the Chern-Lashoff results quoted above, imply Tompkin's theorem [8]: If $k<d$ there are no compact immersions of a locally flat $M^{d}$ in $R^{d+k}$. We now consider the case in which $k=d$.

Received by the editors August 25, 1965. 


\section{Results.}

Theorem. An isometric immersion of $M^{d}$ into $\bar{M}^{2 d}$ which is developable at $m$ in $M$ has all singular second fundamental form operators at $m$ if and only if $\nu(m) \geqq 1$.

Proof. We always assume below that $z$ is in $\bar{M}_{\psi(m)}$ and orthogonal to $d \psi\left(M_{m}\right)$. Clearly if $\nu(m) \geqq 1$ then, since $T_{x} z=S_{z} x$, all $S_{z}$ are singular. Conversely, if all $S_{z}$ are singular we define the function $f(u)$ $=\left\langle T_{u} u, T_{u} u\right\rangle$ where $u$ is in $M_{m}$ and $\langle u, u\rangle=1$. Let $x$ denote a minimum of this function. If this minimum has the value zero then the proof is finished since the developability of $\psi$ implies that for all $u$ in $M_{m}$, $0=\left\langle T_{x} x, T_{u} u\right\rangle=\left\langle T_{x} u, T_{x} u\right\rangle$. We assume $T_{x} x$ is not zero and seek a contradiction.

Case 1. Assume $T_{x}$ is singular. Then for some $u$ in $M_{m}$ orthogonal to $x$ and some number $a, T_{x} u=a T_{x} x$. The function $y(\theta)=x \cos \theta+u \sin \theta$ lies in the domain of $f$ and a simple calculation using the bilinearity of $T$ shows that

$$
(f \circ y)^{\prime}(0)=4\left\langle T_{x} x, T_{x} u\right\rangle .
$$

But since $x$ was a critical point of $f$, this number is zero and hence $a=0, T_{x} u=0$, and therefore $\left\langle T_{x} x, T_{u} u\right\rangle=0$. A second calculation shows

$$
(f \circ y)^{\prime \prime}(0)=4\left\{\left\langle T_{x} x, T_{u} u\right\rangle-\left\langle T_{x} x, T_{x} x\right\rangle+2\left\langle T_{x} u, T_{x} u\right\rangle\right\} \geqq 0,
$$

the inequality following from the minimality property of $x$. Since the first and last terms above are zero, we get the desired contradiction, namely, $T_{x} x=0$.

Case 2. If $T_{x}$ is not singular then there exists a $z$ such that $T_{x} z=b x$ where $b \neq 0$. This implies that $x$ is an eigenvector of the symmetric and singular operator $S_{z}$, and hence that there exists a $u$, orthogonal to $x$ in $M_{m}$, such that $S_{z} u=0$. Now for all $v$ in $M_{m}$ orthogonal to $x$ we have $\left\langle T_{x} x, T_{x} v\right\rangle=0$ since $x$ is a critical point. Hence the $T_{x} v$ span a hyperplane of the $d$-dimensional complement of $d \psi\left(M_{m}\right)$ in $\bar{M}_{\psi(m)}$ and the normal to this hyperplane is $T_{x} x$. Likewise $\left\langle T_{x} v, z\right\rangle=-\left\langle T_{x} z, v\right\rangle$ $=\langle b x, v\rangle=0$. Hence $z$ is parallel to $T_{x} x$ or $z=c T_{x} x$ with $c \neq 0$. Therefore $c\left\langle T_{u} u, \quad T_{x} x\right\rangle=\left\langle T_{u} u, z\right\rangle=-\left\langle T_{u} z, u\right\rangle=0$ since $T_{u} z=0$. Thus $\left\langle T_{u} u, T_{x} x\right\rangle=\left\langle T_{x} u, T_{x} u\right\rangle=0$, or $T_{x} u=0$, a contradiction to the nonsingularity of $T_{x}$. Thus Case 2 cannot occur.

Corollary 1. An isometric immersion of $M^{2}$ into $M^{4}$ has all singular second fundamental form operators at $m$ in $M$ if and only if $\nu(m) \geqq 1$.

Since the difference in the sectional curvatures of a plane $P$ in $M_{m}$ 
and $d \psi(P)$ is just the sum of the determinants of $S_{z}$ and $S_{w}$ where $z$ and $w$ are perpendicular, the singularity of $S_{z}$ and $S_{w}$ imply the developability of the immersion at $m$.

Before giving some implications of this theorem we summarize some definitions and results. Let $N$ denote the bundle of normal $k$ frames of $M^{d}$ relative to $\psi$ : i.e.

$$
\begin{array}{r}
N=\{(m, E) \mid m \in M \text { and } E \text { is a } k \text { frame (orthonormal set of } k \text { vectors) } \\
\text { of } \left.\bar{M}_{\psi(m)} \text { orthogonal to } d \psi\left(M_{m}\right)\right\} .
\end{array}
$$

The Riemannian connection of $\bar{M}^{d+k}$ induces a natural connection on $N$. The curvature form of this connection is called the relative curvature of $\psi,[4]$; it is easy to show that a developable immersion between two manifolds of constant curvature has zero relative curvature at $m$ if and only if all $T_{x}$ commute for all $x$ in $M_{m}$, cf. p. 203 of [4]. Finally $\psi: M^{d} \rightarrow R^{d+k}$ is called $n$-cylindrical provided $M$ and $\psi$ can be expressed as Riemannian products $M^{d}=B^{d-n} \times R^{n}$ and $\psi=\bar{\psi} \times 1$ where $\Psi$ is an isometric immersion of $B^{d-n}$ into $R^{d+k-n}$ and 1 is the identity map of $R^{n}$. Let $M^{d}(C)$ denote a manifold of constant Riemannian curvature $C$. The fundamental result of [5] can be stated in the slightly more general form: If $\psi$ is an isometric immersion from a complete $M^{d}(C)$ into $\bar{M}^{d+k}(C)$ then there exists a totally geodesic complete submanifold of $M$ of dimension equal to the minimum value taken on by the function $\nu$. This submanifold is totally geodesic in $\bar{M}$ relative to $\psi$. Similarly Theorem 3 of [7] states that under the above conditionsif $C>0$; the minimum value of the relative nullity is at least 1 ; and the relative curvature of $\psi$ is zero, then $\psi$ is totally geodesic. Finally Theorem 1 of [7] states that if $\bar{M}^{d+k}(C)=R^{d+k}$ and if $\nu$ is constant on $M$ then $\psi$ is a product immersion.

COROllaRY 2. Every immersion of a complete locally Euclidean $M^{d}$ in $R^{2 d}$ having all singular second fundamental form operators at every point is unbounded.

This is because the image of $\psi$ will contain a line of $R^{2 d}$. Similar results could be stated for the cases where both manifolds are of equal negative or positive curvature; cf. Corollaries 2 and 3 of [5].

CoRollary 3. Every immersion of a locally Euclidean $M^{d}$ in $R^{2 d}$ with all singular second fundamental form operators at each point and constant relative nullity is a product immersion.

Corollary 4. An immersion of $M^{2}(C)$ into $\bar{M}^{4}(C)$ with all singular second fundamental form operators at $m$ in $M$ has zero relative curvature at $m$. 
This follows from the fact that an orthonormal basis $x, y$ of $M_{m}$ can be picked such that $T_{x}=0$; this implies that all the difference operators at $m$ will commute. The immersion of the flat torus in $R^{4}$ as a product of two circles shows that the converse of Corollary 4 is false.

Corollary 5. An immersion of a complete $M^{2}(C)$ into $M^{4}(C)$ with all second fundamental form operators singular everywhere is totally geodesic whenever $C>0$.

\section{REFERENCES}

1. II. Ambrose, The Cartan structural equations in classical Riemannian geometry, J. Indian Math. Soc. 24 (1960), 23-76.

2. S. S. Chern and N. H. Kuiper, Some theorems on the isometric imbedding of compact Riemann manifolds in Euclidean space, Ann. of Math. (2) 56 (1952), 422-430.

3. S. S. Chern and R. K. Lashof, On the total curvature of immersed manifolds, Amer. J. Math 79 (1957), 306-318.

4. B. O'Neill, Isometric immersions of flat Riemannian manifolds in Euclidean space, Michigan Math. J. 9 (1962), 199-205.

5. B. O'Neill and E. Stiel, Isometric immersions of constant curvature manifolds, Michigan Math. J. 10 (1963), 335-339.

6. T. Otsuki, On the existence of solutions of a system of quadratic equations and its geometrical application, Proc. Japan. Acad. 29 (1953), 99-100.

7. E. Stiel, Isometric immersions of manifolds of non-negative constant sectional curvature, Pacific J. Math. 15 (1965), 1415-1419.

8. C. Tompkins, Isometric embedding of fat manifolds in Euclidean space, Duke Math. J. 5 (1939), 58-61.

California State College at Fullerton 